\title{
The performance of DAMPE for $\gamma$-ray detection
}

\author{
Kai-Kai Duan ${ }^{\star a b}$, Yun-Feng Liang ${ }^{a b}$, Zhao-Qiang Shen ${ }^{a b}$, Zun-Lei Xu $\mathbf{u}^{a b}$ and Chuan \\ Yue $^{a b}$ on behalf of the DAMPE Collaboration \\ ${ }^{a}$ Key Laboratory of Dark Matter and Space Astronomy, Purple Mountain Observatory, Chinese \\ Academy of Sciences, Nanjing 210008, China \\ ${ }^{b}$ University of Chinese Academy of Sciences, Beijing 100012, China \\ E-mail: duankk@pmo.ac.cn, xuzl@pmo.ac.cn
}

As one of the important targets of the DArk Matter Particle Explorer (DAMPE), the GeV gammaray observations of DAMPE with good angular resolution, high energy resolution and high background rejection facilitate the search of fine structures in the spectra of gamma-ray sources. In this work we summarize the performance of DAMPE for gamma-ray detection using simulation data, including the effective area, angular resolution and energy resolution.

35th International Cosmic Ray Conference - ICRC2017

10-20 July, 2017

Bexco, Busan, Korea

${ }^{*}$ Speaker. 


\section{Introduction}

The DArk Matter Particle Explorer (DAMPE), is a space mission to measure electron/positron, gamma-ray, proton, helium nuclei and other heavy ions in wide energy ranges with good energy resolution and large acceptance [1,2]. DAMPE is composed of four sub-detectors, a Plastic Scintillator strip Detector (PSD), a Silicon-Tungsten tracKer-converter(STK), a BGO imaging calorimeter and a NeUtron Detector (NUD). The PSD provides charged-particle background rejection for gamma rays (anticoincidence detector) and measures the charge of incident particles; the STK measures the charges and the trajectories of charged particles, and allows to reconstruct the directions of incedent photons converting into $e^{+} e^{-}$pairs; the BGO calorimeter, with a total depth of about 32 radiation lengths, allows to measure the energy of incident particles with high resolution and to provide electron/hadron identification; finally, the NUD provides a further improvement of the electron/hadron identification [1,2].

A full simulation (Monte Carlo) program has been developed to simulate the behavior of detecor response to incident high-energy particles in great detail. The simulation procedure mimics real data production during both round tests and in-flight observation, by using the proper input particle fluxes and fully modeling the detector geometry and readout chain [2]. The MC data can be processed by the same reconstruction algorithms and the DAMPE simulation can provide a accurate representation of the instrument response for analysis. With the on-orbit calibration of trigger threshold, read data trigger logic is then applied to the MC data. All data that pass the trigger forms the final simulation data stream for reconstruction and analysis as it is done for on-orbit data.

The expected instrument performance results from the design of the hardware, the event recontruction and selection algorithms. In principle, the procedure of event reconstruction and selection mainly includes trigger filter, track reconstruction, geometry constraints, charge reconstruction, particle identification and energy reconstruction, any of which has its own efficiency. The efficiency of each block has been carefully studied by using on Monte Carlo simulations and taken into account when evaluating DAMPE's performance. In this paper, the expected performance of DAMPE as a high-energy $\gamma$-ray obsercatory is discussed based on Monte Carlo simulation. The instrument response functions (IRFs), such as effective area, energy and angular resolution, are derived.

\section{Detector Simulation, Event reconstruction and photon selection}

In order to develop the reconstruction and event selection algorithms, we used a detailed simulation of particle interactions with DAMPE. This simulation is based upon the GEANT4 toolkit, a Monte Carlo simulation software widely used in high energy physics experiments, to handle particle generation, propagation and interaction through the instrument with certain physical models. The information of the DAMPE geometry including the position and the material of all the detector elements (both active and passive) is used to build detailed model. A digitzation algorithm has been developed and can be used for converting energy hits into ADC counts which has same format with the raw fight data. To reflect the electronic response of each sub-detector, the digitization algorithm takes into account the on-orbit calibration results such as pedestal noise, PMT gains, non-uniformities etc [2]. 
We simulate uniform $\gamma$-ray fields that we can use to explore the instrument response across the entir FoV and energy range of DAMPE. The $\gamma$ rays are generated with an $E^{-1}$ number spectrum and are randomly generated on a sphere with $6 \mathrm{~m}^{2}$ cross-sectional area centered at the entire DAMPE referance frame. The directions of the $\gamma$ rays are sampled randomly across $2 \pi$ of downward-going directions, so as to respect a semi-isotropic incidence flux.

The event reconstruction algorithm for gamma-rays used here proceeds as follows:

1. Energy reconstruction: The deposited energy in BGO calorimeter cannot be straightforwardly taken as the true energies of incident paticles. The method is used to calculate the corrected energy based on the dependence of deposited energy on some reconstruction variables.

2. Track reconstruction: The BGO track and the STK track are reconstructed respectively first, the direction found in BGO as a seed is formed and track is reconstructed further using the Kalman filter algorithm. The track forks (two tracks starting from the same point) in the direction towards calorimeter are allowed, while those which point in the opposite direction are considered as track crossings and treated correspondingly.

3. Charge reconstruction: For gamma ray detection, PSD serves as an anticoincidence detector (ACD).

Fig. 1 shows the dataflow of detector simulaton and events reconstruction of DAMPE.

DAMPE can measure all particles in case the trigger conditions are met. As well known, the flux of gamma rays is lower than proton flux by 5-6 orders of magnitude. Therefore, resolving gamma rays from cosmic rays is very important for gamma-ray detection of DAMPE. Based on the difference of electromanetic shower and hadronic shower in the BGO calorimeter, We can distinguish the proton from all the particles effectively [3]. As mentioned above, the PSD is used as $\mathrm{ACD}$, its detection efficiency for chared paticles is very powerful for the rejection of cosmic-ray background[3].

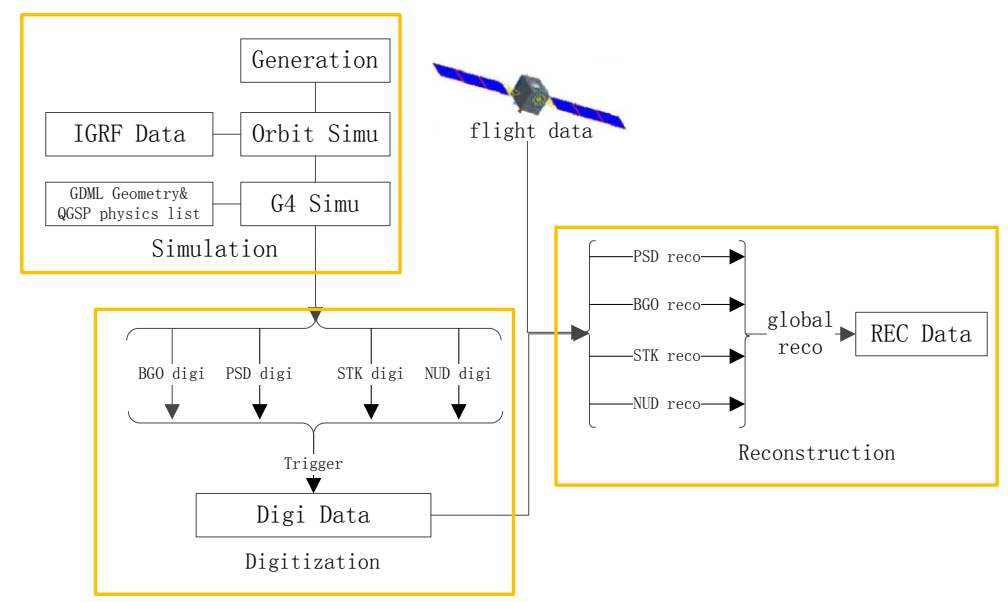

Figure 1: The scheme for DAMPE data simulation and reconstruction (see Fig. 20 in [2]).

\section{Instrument response functions (IRFs)}

\subsection{Effective Area}

In order to correctly evalute the spectra of astrophysical $\gamma$-ray sources, we need to know the 
effective area of DAMPE. In fact, $A_{\text {eff }}$ depends on the geometrical area of DAMPE as well as the efficiency for convering and correctly identifying incident $\gamma$ rays. We use high statistics MC simulations to evaluate $A_{e f f}$. Effective area $A_{e f f}(E, \hat{v}, s)$ : the product of the cross-sectional geometrical collection area, $\gamma$-ray conversion probability, and the efficiency of a given event selection (denoted by $s$ ) for a $\gamma$ ray with energy $E$ and direction $\hat{v}$.

Since the $\gamma$ rays are generated uniformly in $\log (E)$ and solid angle, the effective area in any of the bins, in which the parameter space is partitioned, can be expressed in terms of the total number of generated events $N_{\text {gen }}$ and the number of events $n_{i, j, k}$ passing the $\gamma$-ray selection criteria within the specific bin centered at $E=E_{i}, \theta=\theta_{j}, \phi=\phi_{k}$ :

$$
\begin{gathered}
A_{\text {eff }}\left(E_{i}, \theta_{j}, \phi_{k}\right)=A_{\text {gen }}\left(\frac{n_{i, j, k}}{N_{g e n}}\right)\left(\frac{2 \pi}{\Delta \Omega_{j, k}}\right) \\
\times\left(\frac{\log _{10} E_{\max }-\log _{10} E_{\min }}{\log _{10} E_{\max , i}-\log _{10} E_{\min , i}}\right)
\end{gathered}
$$

The results on effective area is shown in Fig. 2 for normal incidence and 30 degree incidence. The effective area at $100 \mathrm{GeV}$ is about $1200 \mathrm{~cm}^{2}$ for noraml incidence events.

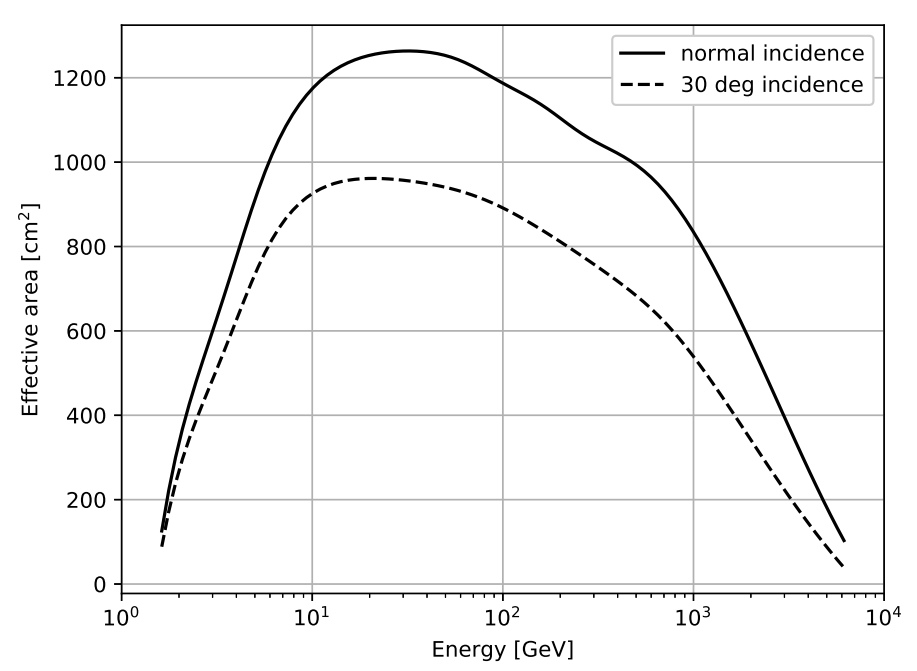

Figure 2: DAMPE effective area for gamma rays. Solid and dashed lines correspond to normal incedence and 30 degree incidence events, respectively (see Fig. 21 in [2]).

\subsection{Angular resolution}

Fig. 3 shows the angular resoluton for gamma rays, which is defined as the separate degree from the incidence that containing $68 \%$ of events. Point-spread function $P\left(\hat{v^{\prime}} ; E, \hat{v}, s\right)$ is the probability density to reconstruct an incident direction $\hat{v}^{\prime}$ for a $\gamma$ ray with $(E, \hat{v})$ in the event selection $s$. The angular resolution is about $0.1^{\circ}$ at $100 \mathrm{GeV}$ for normal incidence events. 


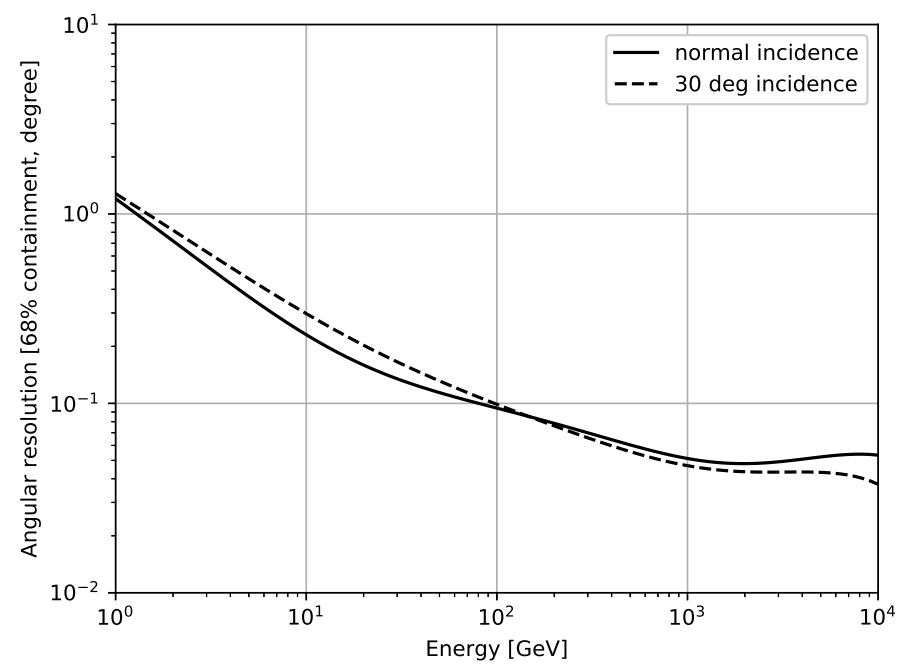

Figure 3: DAMPE angular resolution for gamma rays of normal incidence and 30 degree incidence (see Fig. 24 in [2]).

\subsection{Energy resolution}

Fig. 4 shows the energy resolution for gamma rays of normal incidence and 30 degree incidence. Energy dispersion $D\left(E^{\prime} ; E, \hat{v}, s\right)$ is the probability density to measure an event energy $E^{\prime}$ for $\gamma$ ray with $(E, \hat{v})$ in the event selection $s$. The energy resolution is about $1 \%$ at $100 \mathrm{GeV}$ for normal incidence events.

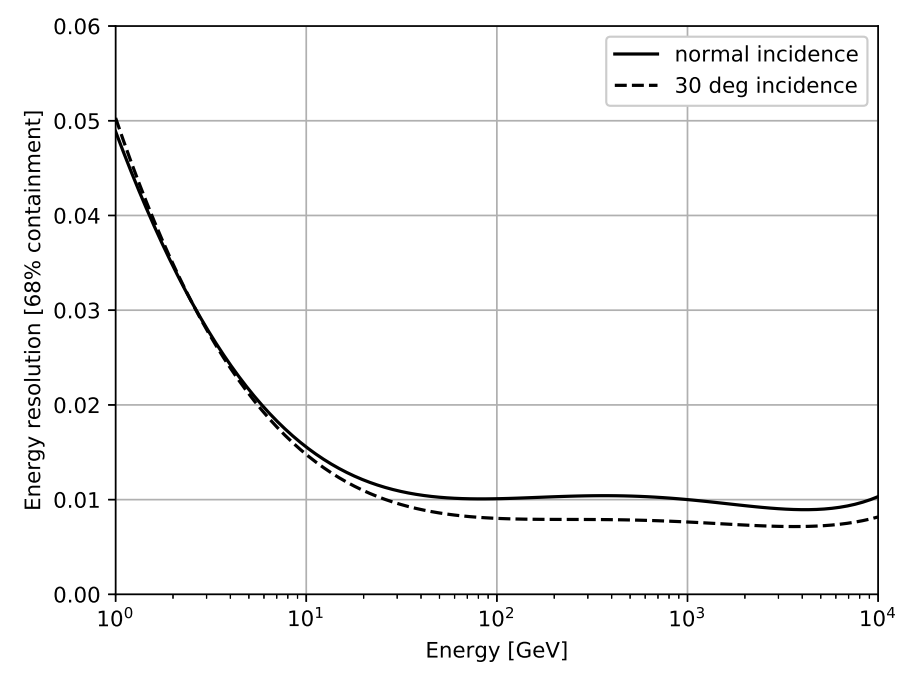

Figure 4: DAMPE energy resolution for gamma rys of normal incidence and 30 degree incidence (see Fig. 23 in [2]). 


\section{Summary}

The performance for the DAMPE for $\gamma$-ray detection has been evaluated with a Monte Carlo simulation, and IRFs are given. Gamma-ray performance of DAMPE are summarized in Table 1. Expected scientific results from gamma-ray observations with this performance will be described in the accompanying paper [4].

Due to its peculiar orbit, the exposure of DAMPE is not uniform over the sky. However, it can cover the all sky every 6 mouths. Thus, DAMPE will be a good all-sky observator of gammarays. Particularly, with its good energy resolution, DAMPE can explore narrow-line feature in the gamma-ray spectrum which might be caused by dark matter annihilation $[5,6]$.

\begin{tabular}{|c|c|}
\hline Parameter & DAMPE \\
\hline Energy range & $5 \mathrm{GeV}-10 \mathrm{TeV}$ \\
\hline Energy resolution at normal incidence & $\sim 1 \% @ 100 \mathrm{GeV}$ \\
\hline Angular resolution at normal incidence & $\sim 0.1^{\circ} @ 100 \mathrm{GeV}$ \\
\hline Effective area at normal incidence & $\sim 1200 \mathrm{~cm}^{2} @ 100 \mathrm{GeV}$ \\
\hline Field of View (FoV) & $\sim 1 \mathrm{sr}$ \\
\hline
\end{tabular}

Table 1: Summary of DAMPE expected performance for gamma-ray detection.

\section{Acknowledgments}

The DAMPE mission was founded by the strategic priority science and technology projects in space science of the Chinese Academy of Sciences (No. XDA04040000 and No. XDA04040400).

In China this work is supported in part by National Key Program for Re- search and Development (No. 2016YFA0400200), the National Basic Research Program (No. 2013CB837000), NSFC under grants No. 11525313 (i.e., Funds for Distinguished Young Scholars), No. 11622327 (i.e., Funds for Excellent Young Scholars), No. XDB23040000, No. 11273070, No. 11303096, No. 11303105, No. 11303106, No. 11303107, No. 11673075, U1531126, U1631111 and the 100 Talents program of Chinese Academy of Sciences. In Europe DAMPE activities are supported by the Italian National Institute for Nuclear Physics (INFN), the Italian University and Research Ministry (MIUR), the Swiss National Science Science Foundation, and the University of Geneva. We also would like to take this opportunity to thank the scientific laboratories and test facilities in China and Europe (in particular the beam test at CERN) that assisted the DAMPE team during the qualification phases.

\section{References}

[1] J. Chang, Dark Matter Particle Explorer: The First Chinese Cosmic Ray and Hard gamma-ray Detector in Space, Chin. J. Spac. Sci., 34550 (2014).

[2] J. Chang, et al. [DAMPE collaboration], The DArk Matter Particle Explorer mission, submitted to Astropart. Phys., arXiv: 1706.08453 (2017).

[3] Z.L. Xu, et al. Gamma-ray selection of DAMPE, in proceedings of ICRC 2017 (2017). 
[4] S.J. Lei, et al. Gamma-ray astronomy with DAMPE, in proceedings of ICRC 2017 (2017).

[5] Y.F. Liang, et al. Search for a gamma-ray line feature from a group of nearby Galaxy clusters with Fermi LAT Pass 8 data, Phys. Rev. D, 93103525 (2016).

[6] Anderson, B. et al. Search for gamma-ray lines towards galaxy clusters with the Fermi-LAT, J. Cosmol. Astropart. Phys., 02026 (2016). 